\title{
Die Bildungsexpansion in der Schweiz - eine Reanalyse unter besonderer Berücksichtigung geschlechtsspezifischer Bildungschancen
}

\section{Christoph Zangger* und Rolf Becker*}

*Universität Bern, Abteilung Bildungssoziologie, CH-3012 Bern, christoph.zangger@edu.unibe.ch und rolf.becker@edu.unibe.ch.

\section{Abstracts \\ Die Bildungsexpansion in der Schweiz - eine Reanalyse unter besonderer Berücksichtigung geschlechtsspezifischer Bildungschancen}

Unter Verwendung der Daten der Schweizer Volkszählungen von 1970 bis 2000 wird der Frage nachgegangen, wie sich die Geschlechterdisparitäten beim Erwerb der Studienzulassung (Maturität) wie auch weiterer Abschlüsse in der Schweiz in und mit der Bildungsexpansion verändert haben. Es kann aus einer handlungstheoretischen Perspektive dargelegt werden, wie sich im betrachteten Zeitverlaufgeschlechtsspezifische Ungleichheiten von Bildungschancen verringern. Die höheren Chancen des Erwerbs der Studienberechtigung gleichen sich für die 18- bis 21-jährigen Frauen ebenso an, wie sich auch deren Wahrscheinlichkeit der Aufnahme einer (dualen) Berufsausbildung erhöht hat. Gleichzeitig erlaubt die Differenzierung der untersuchten Abschlüsse im Vergleich zu vorangegangenen Untersuchungen präzisere Aussagen zur Entwicklung weiterer herkunftsbedingter Ungleichheiten.

Schlüsselwörter: Bildungsexpansion, Bildungschancen, geschlechtsspezifische Bildungsentscheidungen, Schweiz, Volkszählung

L'expansion du système de formation en Suisse: une nouvelle analyse tenant particulièrement compte des chances de formation en fonction du sexe

Sur la base des données recueillies lors des recensements de la population suisse de 1970 à 2000, nous avons cherché à savoir comment les disparités entre sexes avaient évolué en matière d'accès aux études (maturité) et d'obtention d'autres diplômes en Suisse à travers l'expansion du système de formation. En se basant sur une approche théorique de l'action, il est possible d'observer la diminution des inégalités entre les sexes face aux chances de formation durant cette période. Les femmes de 18 à 21 ans ont désormais presque les mêmes chances que les hommes d'accéder aux études et leur probabilité d'être admises à une formation professionnelle (duale) a également augmentée. La différenciation des formations permet - à travers la comparaison avec des analyses précédentes - des déclarations plus précises sur le développement des inégalités sociales.

Mots-clés: Expansion du système de formation, chances de formation, choix de formation spécifiques au sexe, Suisse, recensement de la population

\section{Educational Expansion in Switzerland - A Reanalysis with Special Consideration of Gender}

Using data from the Swiss census from 1970 to 2000, this contribution investigates how gender-specific disparities in acquiring the high school diploma (Matura) and therewith the admission to university, as well as other diplomas changed in and with the educational expansion in Switzerland. Thereby, the decision-theoretic extension of a recent contribution is taken as a starting point. During the period under study, gender-specific inequalities declined. At the same time as the gender gap with higher chances to obtain a high school diploma for young women aged 18 to 21 decreased, these women also became more likely to participate in vocational education, previously dominated by men. Furthermore, the differentiation of additional levels of educational attainment compared to prior studies also allows us to make more precise statements about the development of inequalities due to social origin. Keywords: Educational expansion, educational opportunities, gender-specific educational decisions, Switzerland, census 


\title{
Die Bildungsexpansion in der Schweiz - eine Reanalyse unter besonderer Berücksichtigung geschlechtsspezifischer Bildungschancen
}

\author{
Christoph Zangger* und Rolf Becker*
}

\section{Ausgangspunkt}

Einer Studie von Becker und Zangger (2013) zufolge führte die Bildungsexpansion in der Schweiz zur Höherqualifikation in der Bevölkerung, von der vor allem Mädchen und Frauen in den jüngeren Geburtskohorten profitierten. Der vorliegende Beitrag nimmt diese Studie als Ausgangspunkt und vertieft die von den Autoren angesprochene - und durch historische und international vergleichende Studien empirisch unterstützte - Annahme, dass Mädchen und Frauen als Folge verbesserter Bildungsgelegenheiten (z. B. Ausbau von Gymnasien) und ihrer ohnehin schon besseren schulischen Leistungen (z. B. bessere Notendurchschnitte und höhere Erfolgsaussichten) ihre Rückstände beim Zugang zu höherer Bildung gegenüber Knaben und Männern mehr als nur ausgeglichen haben (Pfeffer 2008; Breen et al. 2010; Hadjar und Berger 2010; Breen et al. 2012; Becker et al. 2013; DiPrete und Buchmann 2013; Becker 2014). Bereits vorliegende Forschungsergebnisse über Bildungsverläufe legen dabei nahe, dass Mädchen im Vergleich zu gleichaltrigen Knaben zwar immer schon eher die Maturitätsschule bzw. das Gymnasium besucht haben und eher die Studienberechtigung (Maturität) erworben haben (vgl. Rieger 2001; Becker und Zangger 2013), jedoch in der Vergangenheit vermutlich seltener ihre Bildungslaufbahn an Universitäten und in der höheren Berufsbildung fortgesetzt haben (Buchmann und Charles 1993; Buchmann et al. 2007; Becker 2012; Glauser und Becker 2015). Dieser Heterogenität geschlechterspezifischer Bildungslaufbahnen wird jedoch in der Analyse von Becker und Zangger (2013) nur bedingt Rechnung getragen.

Einerseits kann auf Basis der Volkszählungsdaten keine adäquate Modellierung der weiteren Bildungsverläufe sowohl von Männern als auch von Frauen nach dem Erwerb der Hochschulzulassung erfolgen und somit fehlt dann auch eine - dem Anliegen der Autoren korrespondierende - umfassende Darstellung der Entwicklung ab diesem Zeitpunkt für die Schweiz (Franzen et al. 2004; Hadjar und Berger 2010). Wird jedoch die amtliche Statistik herangezogen, so lässt sich auf der aggregierten Ebene für die Schweiz ein Bild zeichnen, nach welchem junge Frauen in den letzten

Universität Bern, Abteilung Bildungssoziologie, CH-3012 Bern, christoph.zangger@edu.unibe.ch und rolf.becker@edu.unibe.ch. 
Jahren nicht nur aufgeholt, sondern die gleichaltrigen Männer sowohl hinsichtlich des Erwerbs der Maturität als auch der effektiven Aufnahme eines Studiums hinter sich gelassen haben (Becker et al. 2013). So lag im Jahre 2010 die Studienanfängerquote für Frauen bei 39\% und für Männer jedoch «nur» bei 34\%. Zum Vergleich dazu: Ende der 1990er Jahre nahmen 27\% der Männer und lediglich 18\% der Frauen der jeweiligen altersgleichen Referenzpopulation ein Studium auf. Andererseits muss eine Modellierung geschlechtssegregierter Bildungsverläufe auch deren Heterogenität adäquat widerspiegeln. Hierbei wird eine binäre Modellierung geschlechtsspezifischer Chancen, eine Hochschulzugangsberechtigung zu erwerben, der Diversifizierung des schweizerischen Bildungssystems nicht gerecht, werden doch in der Referenzkategorie zur Maturität eine Reihe unterschiedlicher Abschlüsse zusammengefasst (z. B. weitere allgemeinbildende wie auch berufsbildende Bildungsgänge). ${ }^{1}$ Gerade aus entscheidungstheoretischer Sicht des Individuums sowie aus institutioneller Sicht des differenzierten Bildungssystems ist die simultane Berücksichtigung der zur Verfügung stehenden Alternativen jedoch essenziell (Schimpl-Neimanns 2000, 649; Becker 2000). Unter Vernachlässigung weiterer Bildungsoptionen in Modellschätzungen können die tatsächliche Entscheidungssituation sowie die damit verbundenen Einflussfaktoren und Folgen für den Bildungsverlauf somit nicht adäquat abgebildet werden (vgl. Breen und Jonsson 2000).

Eben diesem Anliegen nimmt sich der vorliegende Beitrag an und erweitert somit nicht nur in methodischer, sondern auch in inhaltlicher Hinsicht vorangegangene Arbeiten zur Bildungsexpansion bzw. deren Verlauf und Auswirkungen in der Schweiz. Wiederum werden die Datensätze der harmonisierten Volkszählung (VZ) aus den Jahren 1970, 1980, 1990 und 2000 herangezogen (Busset 1993; Sheldon 2005; Becker und Zangger 2013, 433 ff.). ${ }^{2}$ Die Analysen beschränken sich wiederum auf Frauen und Männer im Alter von 18 bis 21 Jahren, die noch im Elternhaushalt wohnhaft sind, da nur für diese Personen die soziale Herkunft nach Klassenlage und höchster Bildung der Eltern sowie der Migrationshintergrund rekonstruiert werden

1 Indes birgt die Harmonisierung der Volkszählungsdaten über die vier Messzeitpunkte hinweg nicht nur methodische, sondern auch inhaltliche Herausforderungen. Beispielsweise ist eine Differenzierung der 1994 eingeführten Berufsmaturität, welche zum Besuch der Fach-, nicht jedoch der universitären Hochschulen berechtigt, von der allgemeinbildenden Maturität nicht möglich. Somit werden jedoch gerade auch für die Beantwortung der Frage nach den Folgen der Bildungsexpansion relevante Aspekte der durch selbige angeleitete Prozesse des Wandels im schweizerischen Bildungssystem erschwert untersuchbar.

2 In den Volkszählungsdaten werden bis in das Jahr 2000 alle Haushalte bzw. Personen erfasst. Dabei werden neben haushaltsbezogenen Charakteristika insbesondere auch Individualmerkmale wie etwa das Geschlecht, Geburtsland, Ausbildung, Beruf bzw. berufliche Stellung und die Stellung im Haushalt erhoben. Diese Merkmale ermöglichen es somit, die Bildungsexpansion für das 20 . Jahrhundert in der Schweiz nachzuzeichnen. Indes wäre eine Weiterführung der Analysen unter Verwendung aktueller Daten der Strukturerhebung ab dem Jahr 2010 wünschenswert. Obschon diese Daten auch den Autoren zur Verfügung stehen, ist dieses Anliegen jedoch aus datentechnischen Gründen nicht realisierbar, weil die für die vorliegende Analyse notwendige Rekonstruktion erworbener Bildungsabschlüsse aller Haushaltsmitglieder (insbesondere Eltern und deren noch im Elternhaus lebenden Kinder) nicht wie bei den harmonisierten Volkszählungen möglich ist. 
kann. Auf dieser Basis versuchen wir, die Frage zu klären, wie sich die geschlechtsspezifischen Chancen für den Erwerb unterschiedlicher Bildungsabschlüsse nach der obligatorischen Schulzeit auf der Sekundarstufe II im Zeitverlauf gewandelt haben. Die dabei gewonnen Erkenntnisse werden mit früheren Ergebnissen kontrastiert und bilden somit eine Erweiterung wie auch Replikation der allgemein gehaltenen Studie von Becker und Zangger (2013).

Um diesem Anliegen gerecht zu werden, unterscheiden wir mit der abhängigen Variable nicht lediglich dichotom zwischen dem Erreichen bzw. Nichterreichen der Maturität, sondern erweitern sie um die im Bildungssystem tatsächlich zur Disposition stehenden Alternativen einer berufs- und einer allgemeinbildenden Ausbildung. Die abhängige Variable - der höchste erreichte Abschluss im Alter zwischen 18 und 21 Jahren - umfasst somit drei Ausprägungen: 1) maximal Diplommittelschule oder vergleichbare allgemeinbildende Bildungsgänge (inklusive Abschluss der obligatorischen Schule), 2) Berufsausbildung, wobei hier auf Grund des zur Verfügung stehenden Datenmaterials sowohl Berufslehren wie auch Vollzeitberufsschulen zusammengefasst werden, und 3) die Maturität. Auf eine weitere Berücksichtigung der Abschlüsse der höheren Berufsbildung und der Fachmittelschulen muss einerseits hinsichtlich der geringen Zellbesetzung (weniger als 1\%) und der damit verbundenen Schätzprobleme (Long 1997), andererseits auf Grund der für den Vergleich über den Zeitverlauf vorgenommenen Harmonisierung der Daten durch das Bundesamt für Statistik verzichtet werden. ${ }^{3}$

\section{Empirische Ergebnisse}

Nachfolgend berichten wir - vergleichend mit der ursprünglichen Analyse von Becker und Zangger (2013) - die Ergebnisse einer differenzierten Betrachtung bislang erreichter Bildungsabschlüsse für die 18- bis 21-jährigen, noch im Elternhaushalte lebenden Personen unter besonderer Berücksichtigung geschlechtsspezifischer Effekte über den Zeitverlauf. Wir konzentrieren uns dabei im Wesentlichen auf die Herausarbeitung der Gemeinsamkeiten und Unterschiede in den Ergebnissen und verzichten auf eine ausführliche Darstellung der allgemeinen, bereits anderweitig dargestellten Entwicklung der Bildungsexpansion in der Schweiz.

\subsection{Entwicklung der untersuchten Bildungsabschlüsse im Zeitverlauf}

Wie in Abbildung 1 ersichtlich, lassen sich für die drei interessierenden Bildungsabschlüsse (Maturität, Berufsbildung und obligatorische bzw. Diplommittelschule) jeweils deutliche Trends über den Zeitverlauf (historische Periode und Geburtskohorten) ausmachen. Indes kann bereits vorweg genommen werden, dass sich ein beacht-

3 So kann aus diesem Grund auch nicht zwischen dem Abschluss der obligatorischen Schule und dem weiteren Besuch einer Diplommittelschule unterschieden werden. 


\section{Abbildung 1 Bildungsabschlüsse im Zeitverlauf - nur 18- bis 21-jährige Frauen und Männer}
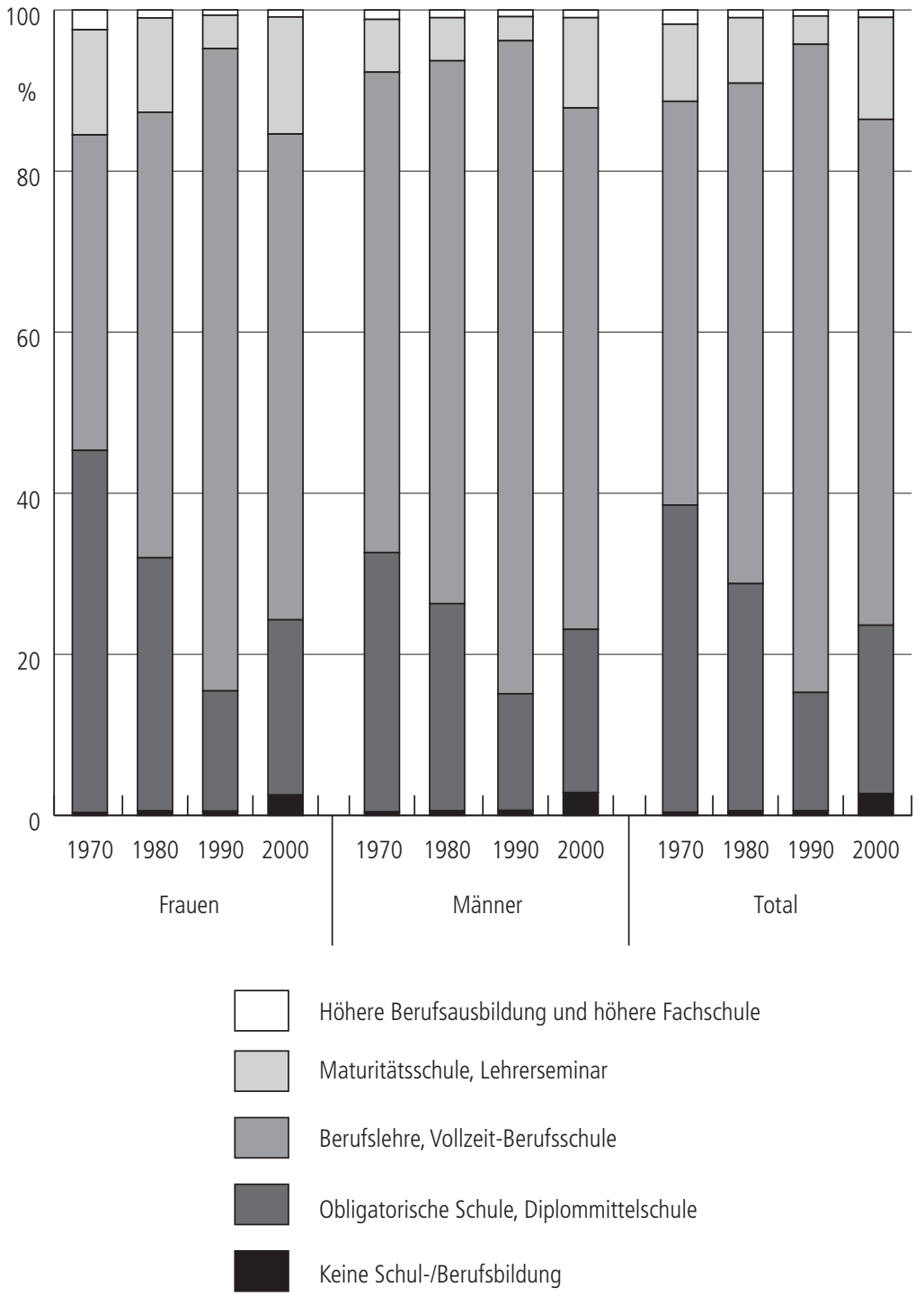

N Insgesamt: 114197 (1970), 112592 (1980), 102267 (1990), 70350 (2000).

N Frauen: 53011 (1970), 49100 (1980), 46090 (1990), 30849 (2000).

N Männer: 61186 (1970), 63492 (1980), 56177 (1990) 39501 (2000).

Quelle: Harmonisierte Volkszählungen der Schweiz - eigene Berechnungen. 
licher Teil der 18- bis 21-Jährigen zu den Messzeitpunkten in Ausbildung befindet (Im Vergleich dazu: 1970 waren es noch 39.6\% und im Jahre 2000 befanden sich bereits 65.2\% dieser Altersgruppe in einer Ausbildung). Dieser Umstand alleine kann bereits als eindrücklicher Hinweis auf die Folgen der Bildungsexpansion - längere Verweildauer im Bildungssystem und damit verbundener Höherqualifikation (vgl. Becker und Zangger 2013) - herangezogen werden und erklärt beispielsweise auch die vergleichsweise geringe Maturitätsquote von rund 10\% pro Geburtsjahrgang. Andererseits stellt sich in gerechtfertigter Weise auch die Frage, ob hiermit nicht Schlussfolgerungen auf Basis eines selektiven Samples gezogen werden. Hierzu ist jedoch anzuführen, dass diese Verzerrung eine Interpretation der nachfolgenden Effekte als «untere Grenze» des Einflusses der Bildungsexpansion nahelegt, werden doch lediglich diejenigen Personen einbezogen, die sich nicht in einer weiterführenden Ausbildung befinden.

Hinsichtlich der höchsten abgeschlossenen Ausbildung beobachten wir zunächst bei der obligatorischen Schulbildung (als «Mindestbildung») und weiterer allgemeinbildender Abschlüsse wie der Diplommittelschule eine Angleichung zwischen den Geschlechtern. Während 1970 noch rund 45\% der Frauen und «lediglich» gut 32\% der Männer über diesen Abschluss verfügten, so lagen die jeweiligen Anteile im Jahr 2000 bei vergleichbaren 21\% bzw. 20\%. Diese Entwicklung kann wiederum als eine wichtige Grundlage für die nachhaltige Bildungsexpansion in der Schweiz angesehen werden. Weiter ist für die Berufsbildung hervorzuheben, dass im untersuchten Zeitraum eine deutliche Zunahme der Beteiligung der Frauen an selbiger stattgefunden hat. Zwischen 1970 und 1990 stieg der Anteil der 18- bis 21-jährigen Frauen mit diesem Bildungsniveau von 39\% auf rund 80\% und ging dann bis 2000 wieder auf - einen mit den Männern vergleichbaren Wert von - gut 60\% zugunsten der höheren Schulbildung zurück.

Bezüglich des dritten zu untersuchenden Abschlusses, der Maturität, scheint die Entwicklung auf den ersten Blick etwas unerwartet. Zwar wird einerseits dargelegt, dass bereits 1970 deutlich mehr Frauen als Männer die Maturität als höchsten Bildungsabschluss aufwiesen (13\% vs. weniger als $7 \%$ ) und über die Zeitpunkte eine Angleichung zwischen den Geschlechtern stattfindet (2000: 14\% vs. 11\%), andererseits überrascht der deutliche Rückgang zum Messzeitpunkt 1990 für beide Geschlechter. Dieses Faktum kann weder auf eine Selektivität in der untersuchten Gruppe aufgrund des späteren Auszuges aus dem Elternhaus als Folge der Bildungsexpansion (Hill und Kopp 2013) noch auf strukturelle Prozesse der Bildungsexpansion - also auf die längere Verweildauer im Bildungssystem - selbst zurückgeführt werden. Eine empirisch abgesicherte Erklärung muss auf Grund des zur Verfügung stehenden Datenmaterials offen bleiben. Es sei lediglich angemerkt, dass es sich um einen für diese Altersgruppe spezifischen "Einbruch" zu handeln scheint, weist doch etwa die Kohorte der 30-39 Jährigen im Jahr 2000 auf keinen 
solchen systematischen Rückgang hin (vgl. Abbildung 2 in Becker und Zangger 2013; siehe auch Jann und Combet 2012).

\subsection{Multivariate Analysen zur sozialen Ungleichheit von Bildungschancen}

Wie haben sich die relativen Effekte herkunftsbedingter und geschlechtsspezifischer Bildungsentscheidungen während der Bildungsexpansion verändert? Um diese Frage zu beantworten, wird die relative Chance geschätzt, maximal mit dem obligatorischen Schul- bzw. dem Diplommittelschulabschluss oder mit der (dualen bzw. vollzeitschulischen) Berufsausbildung im Verhältnis zum Erwerb der (gymnasialen) Maturität abzuschliessen. Die Schätzung erfolgt mittels multinomialer logistischer Regression (vgl. Long 1997). Die Wahl dieses Schätzverfahrens erfolgt einerseits aus methodischen, andererseits aus inhaltlichen Gründen für eine adäquate Abbildung von Bildungsentscheidungen im Sinne einer strukturell-individualistischen Erklärung (vgl. Urban 1993; Becker 2000).

Die geschätzten Koeffizienten werden als Average Marginal Effects (AME) einer Masszahl für die «mittlere» Stärke des Effekts auf die Wahrscheinlichkeit, einen bestimmten Bildungsabschluss zu erwerben - dokumentiert. Die AME erlauben den Vergleich der Koeffizienten über Messzeitpunkte und Gruppen hinweg (vgl. Allison 1999; Hinz und Auspurg 2011; Best und Wolf 2012). Wegen geringer Zellbesetzungen bei der multinomialen Regression wird bei der Messung des Migrationshintergrunds nach Herkunftsland keine Differenzierung nach der Generation vorgenommen. Dafür wird zusätzlich die Sprache im Elternhaus berücksichtigt. Die soziale Herkunft wird sowohl über das höchste Bildungsniveau der Eltern als auch über die Klassenlage des Elternhauses nach dem Klassenschema von Erikson und Goldthorpe (1992) kontrolliert (vgl. auch Bukodi und Goldthorpe 2013). Hierbei werden zusätzlich Versorgungsklassen berücksichtigt, die ihren Lebensunterhalt durch staatliche Transfereinkommen bestreiten (vgl. Lepsius 1979).

Wie die deskriptive Darstellung in Abbildung 1 bereits vermuten liess, haben bei Kontrolle der sozialen und ethnischen Herkunft die 18- bis 21-jährigen Frauen im Vergleich zu gleichaltrigen Männern in der Tat eine höhere Chance, die (gymnasiale) Maturität zu erwerben (Tabelle 1). Beim Erwerb der gymnasialen Maturität haben sich geschlechtstypische Ungleichheiten im Zeitverlauf tendenziell verringert. Deutlich ausgeprägter verläuft diese Angleichung bei der Berufsausbildung. Nach wie vor entscheiden sich Männer zwar häufiger als Frauen für diese Option. Jedoch verringerte sich der geschlechtsspezifische Effekt im betrachteten Zeitraum auf knapp einen Viertel seiner ursprünglichen Grösse. Am deutlichsten zeigen sich die Effekte der Bildungsexpansion auf die Geschlechterunterschiede beim Erwerb der obligatorischen Schulausbildung und dem Besuch einer allgemeinbildenden Schule wie der Diplommittelschule. Während in der bivariaten Darstellung in Abbildung 1 bereits ab 1980 kaum noch Unterschiede zwischen Frauen und Männern beim Erwerb dieser Abschlüsse festzustellen sind, zeichnet die multivariate Analyse eine 
noch deutlichere Entwicklung hin zum Ausgleich der ursprünglich prägnanten Unterschiede. Während 1970 für 18- bis 21-jährige Frauen die Wahrscheinlichkeit durchschnittlich gut 15\% höher war, sich für den Verbleib mit einem obligatorischen Schulabschluss oder einer Diplommittelschule zu entscheiden, reduzierte sich dieser Geschlechtereffekt im Jahre 2000 auf rund 1.7\%. Diese komplementäre Entwicklung in den drei untersuchten Ausbildungsoptionen unterstreicht gleichzeitig die Adäquanz der Modellierung aller relevanten Bildungsalternativen. So zeigt sich dann auch deutlich, dass gerade die Entwicklung der geschlechtsspezifischen Unterschiede im Vergleich zur ursprünglichen Analyse von Becker und Zangger (2013) deutlicher zutage treten, werden doch in der ursprünglichen Referenzkategorie zwei entgegengesetzte Effekte zusammengefasst.

Indes erweist sich die vorgenommene Differenzierung der abhängigen Variable jedoch auch für die Entschlüsselung der Folgen der Bildungsexpansion - nämlich für die Entwicklung herkunftsbedingter Ungleichheiten - als äusserst aufschlussreich. Während sich auch in der vorliegenden Analyse das Bild eines reduzierten Einflusses der sozialen Herkunft bis 1990 und einer Zunahme bis ins Jahr 2000 für den Erwerb der Matura ergibt, so wird dieses durch einen persistenten Effekt der sozialen Herkunft auf den Abschluss einer Berufsausbildung ergänzt. Insbesondere ist bemerkenswert, wie für diesen Abschluss einerseits der Einfluss der sozialen Herkunft (gemessen an der elterlichen Bildung und deren Klassenposition) im Zeitverlauf leicht zunimmt und andererseits der Effekt von Letzterer unter Kontrolle der elterlichen Bildung und allen weiteren Kovariaten das Vorzeichen wechselt. Nicht nur bleiben diese Effekte auf Grund des Designs in der ursprünglichen Studie von Becker und Zangger (2013) unberücksichtigt, sondern sie unterstreichen gleichzeitig auch eine weitere Konsequenz der Bildungsexpansion: Während 1970 und 1980 Angehörige der unteren und oberen Dienstklasse unter expliziter Kontrolle der elterlichen Bildung noch häufiger in die Berufsbildung wechselten als Kinder aus den tieferen Sozialschichten, haben sich die Verhältnisse im Jahr 2000 umgekehrt. Somit scheinen die 18- bis 21-jährigen Frauen und Männer aus den privilegiertesten Schichten heutzutage verstärkt von einer Berufsausbildung zu Gunsten der allgemeinen Maturität oder aber auch der Diplom- und allgemeinbildenden Schulen wie der Diplommittelschule abzusehen.

Dieser Wandel ist nicht bloss Ausdruck einer mit der Bildungsexpansion einhergehenden Tertiarisierung der Erwerbstätigkeit, welche mit einer zunehmenden Integration von Frauen in das System höherer Bildung und Beschäftigung im Dienstleistungsbereich und somit - langfristig gesehen - zum Abbau geschlechtstypischer Ungleichheiten in weiteren gesellschaftlichen Teilbereichen beiträgt (vgl. DiPrete und Buchmann 2013; Becker 2014), sondern gerade der Umstand, dass die Kinder aus den Dienstklassen bei gleichem Bildungsstand der Eltern zunehmend von der Berufsausbildung absehen, zeigt deutlich, wie im Zuge der Bildungsexpansion Ungleichheiten nicht einfach nivelliert werden, sondern als Konsequenz neue 


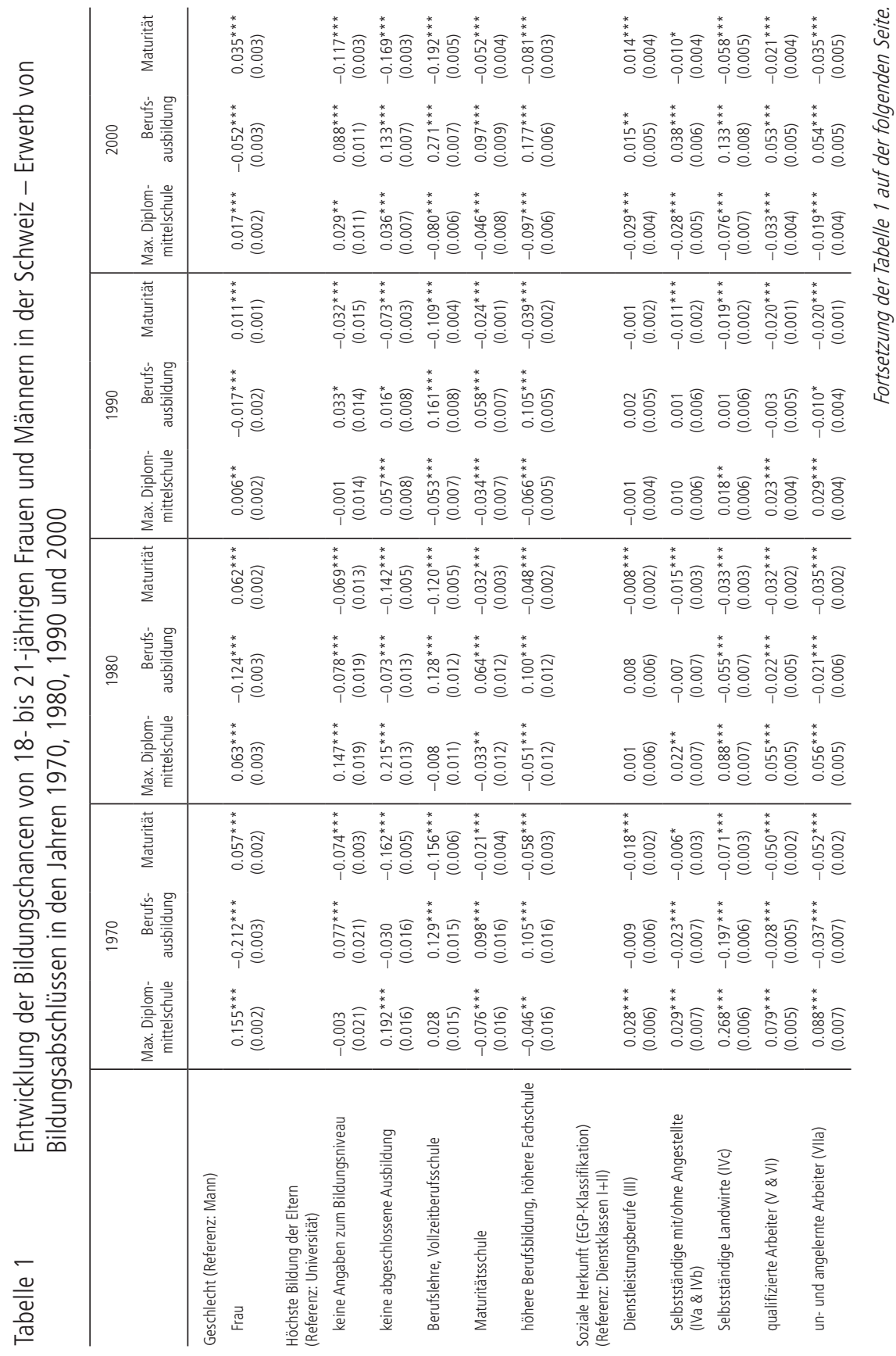




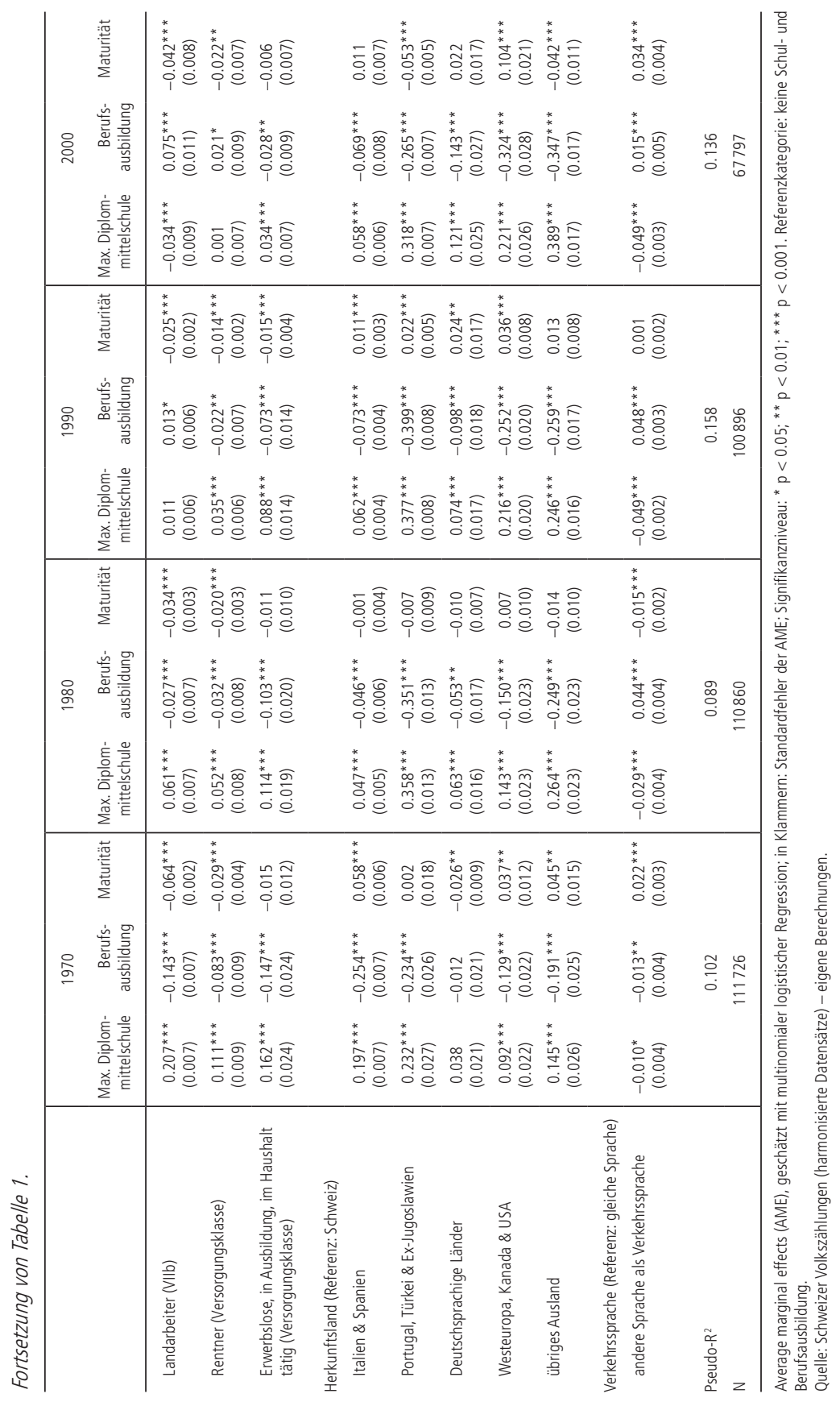


Einschätzungen der Instrumentalität unterschiedlicher Bildungsabschlüsse zustande kommen und entsprechend neue - mitunter ungleichheitserhaltende - Bildungsentscheidungen getroffen werden.

\section{Zusammenfassung und Schlussfolgerung}

Ziel der vorliegenden Reanalyse ist es, die Befunde der Studie von Becker und Zangger (2013) unter besonderer Berücksichtigung geschlechtsspezifischer Bildungschancen im Zuge der Bildungsexpansion in der Schweiz nach Mitte des 20. Jahrhunderts nachzuzeichnen. Mittels der Schweizer Volkszählungsdaten werden die Chancen, die gymnasiale Maturität zu erreichen im Vergleich zu anderen Bildungsabschlüssen untersucht. Hierbei wurde im Unterschied zu einer früheren Analyse aus theoretischen und methodischen Gründen die abhängige Variable in ihrer initialen Kodierung belassen. Somit waren multinomiale Schätzungen möglich. Die Befunde zeigen, dass 18- bis 21-jährige, noch im Elternhaus lebende Frauen im gesamten betrachteten Zeitraum im Vergleich zu altersgleichen Männern günstigere Chancen haben, die Maturität zu erlangen. Allerdings sind im betrachteten Zeitraum von 1970 bis 2000 die Unterschiede zwischen den Geschlechtern zurückgegangen - insbesondere beim Erwerb einer Berufsausbildung und dem Abschluss der obligatorischen oder Diplommittelschule.

Von einem grundlegenden Rückgang der Bildungsungleichheiten nach Geschlecht, aber auch nach sozialer Herkunft und Migrationshintergrund im Zuge der Bildungsexpansion zu sprechen, wäre angesichts der desolaten Datenlage für die Bildungsforschung in der Schweiz vermessen. Gerade die Kontrastierung des leichten Rückganges der Ungleichheit nach sozialer Herkunft beim Erwerb der Maturität bis zum Messzeitpunkt 1990 durch eine stagnierende bis sogar zunehmende Ungleichheit hinsichtlich des Abschlusses einer Berufsausbildung sowie die Tatsache, dass sich Angehörige der Dienstklassen bei gleichem Bildungshintergrund wie die übrigen Sozialschichten im Jahr 2000 (im Gegensatz zu 1970 und 1980) häufiger gegen eine Berufsausbildung entscheiden, verweist auf den Umstand, dass bestehende Bildungsungleichheiten im Zuge der Bildungsexpansion nicht nivelliert worden sind. Dieser letzte Punkt wirft zusätzlich die Frage auf, ob als Konsequenz der Bildungsexpansion selbst sich die individuelle Evaluation der einzelnen Bildungstitel und ihrer Instrumentalität insofern geändert hat, als dass daraus sogar wiederum eine Verstärkung der Ungleichheit - zumindest nach sozialer Herkunft - eintritt (vgl. Müller 1998). Allerding kann diese Frage weder auf Basis der dieser Studie zugrunde liegenden Volkszählungsdaten noch mit anderen für die Sozialwissenschaften zugänglichen Statistiken für einen längeren historischen Zeitraum und aufeinander folgende Geburtskohorten sowie mit hinreichend grossen Fallzahlen bis in die jüngste Gegenwart beantwortet werden (vgl. Jann und Combet 2012). 
Andere Datensätze - wie etwa das Schweizerische Haushaltspanel oder die Schweizerische Arbeitskräfteerhebung - sind aufgrund geringer Fallzahlen nur bedingt aussagekräftig, wenn es um Effekte sozialer und ethnischer Herkunft für Bildungschancen sowie um geringfügige Veränderungen im geschlechtsspezifischen Bildungsverhalten und bei Bildungsergebnissen geht (Becker und Zangger 2013, 426). Gleiches gilt für die Panelstudie TREE (Scharenberg et al. 2014). Um die methodischen Unzulänglichkeiten der bislang verfügbaren Quer- und Längsschnittdaten auszugleichen, werden lückenlose Längsschnittdaten im Kohortendesign und mit hohen Fallzahlen - etwa nach dem Vorbild der Deutschen Lebensverlaufsstudie (Mayer 2008) oder des Nationalen Bildungspanels (Blossfeld et al. 2011) - benötigt. Schliesslich müssen auch dann, und das ist aus der Reanalyse zu lernen, die Bildungsabschlüsse möglichst differenziert abgebildet werden, wenn es um geschlechtsspezifische Bildungschancen und den Geschlechtervergleich beim Erwerb von Bildungszertifikaten im Zeitverlauf geht (Müller und Klein 2008).

\section{$4 \quad$ Literaturverzeichnis}

Allison, Paul D. 1999. Comparing logit and probit coefficients across groups. Sociological Methods \& Research 28(2): 186-208.

Becker, Rolf. 2000. Klassenlage und Bildungsentscheidungen. Eine empirische Anwendung der WertErwartungstheorie. Kölner Zeitschrift für Soziologie und Sozialpsychologie 52(3): 450-475.

Becker, Rolf. 2012. Der Übergang ins Hochschulstudium: Prozesse und Mechanismen am Beispiel der deutschen Schweiz. S. 305-331 in Bildung - Arbeit - Erwachsenwerden. Ein interdisziplinärer Blick auf die Transition im Jugend- und jungen Erwachsenenalter, hrsg. von Max Bergman, Sandra Hupka-Brunner, Thomas Meyer und Robin Samuel. Wiesbaden: VS Verlag für Sozialwissenschaften.

Becker, Rolf. 2014. Reversal of gender differences in educational attainment - historical analysis of the West German case. Educational Research 56(2): 184-201.

Becker, Rolf, Michael Beck und Franziska Jäpel. 2013. Geschlechterunterschiede im Bildungserfolg. Eine empirische Analyse für Schulkinder im Deutschschweizer Schulsystem unter besonderer Berücksichtigung ihres Migrationshintergrundes. S. 77-101 in Geschlecht, Migrationshintergrund und Bildungserfolg, hrsg. von Andreas Hadjar und Sandra Hupka-Brunner. Weinheim/München: Juventa.

Becker, Rolf und Christoph Zangger. 2013. Die Bildungsexpansion in der Schweiz und ihre Folgen. Eine empirische Analyse des Wandels der Bildungsbeteiligung und -ungleichheiten mit den Daten der Schweizer Volkszählungen 1970, 1980, 1990 und 2000. Kölner Zeitschrift für Soziologie und Sozialpsychologie 65(3): 423-449.

Best, Henning und Christof Wolf. 2012. Modellvergleich und Ergebnisinterpretation in Logit- und Probit-Regressionen. Kölner Zeitschrift für Soziologie und Sozialpsychologie 64(2): 377-395.

Blossfeld, Hans Peter, Jutta von Maurice und Thorsten Schneider. 2011. Education as a lifelong process. Zeitschrift für Erziehungswissenschaft 11 (Sonderheft 14): 19-34.

Breen, Richard und Jan O. Jonsson. 2000. Analyzing educational careers: A multinomial transition model. American Sociological Review 65(5): 754-773. 
Breen, Richard, Ruud Luijkx, Walter Müller und Reinhard Pollak. 2010. Long-term trends in educational inequality in Europe: Class inequalities and gender differences. European Sociological Review 26(1): 31-48.

Breen, Richard, Ruud Luijkx, Walter Müller und Reinhard Pollak. 2012. Bildungsdisparitäten nach sozialer Herkunft und Geschlecht im Wandel - Deutschland im internationalen Vergleich. S. 346-373 in Soziologische Bildungsforschung, hrsg. von Rolf Becker und Heike Solga. Sonderheft 52 der Kölner Zeitschrift für Soziologie und Sozialpsychologie. Wiesbaden: Springer VS.

Buchmann, Marlis und Maria Charles. 1993. The lifelong shadow: Social origins and educational opportunities in Switzerland. S. 177-192 in Persistent inequalities. Changing Educational Stratification in Thirteen Countries, hrsg. von Yossi Shavit und Hans-Peter Blossfeld. Boulder, CO: West View Press.

Buchmann, Marlis, Stefan Sacchi, Markus Lamprecht und Hanspeter Stamm. 2007. Tertiary education expansion and social inequality in Switzerland. S. 321-348 in Stratification in Higher Education, hrsg. von Yossi Shavit, Richard Arum und Adam Gomoran. Stanford: Stanford University Press.

Bukodi, Erzebet und John H. Goldthorpe. 2013. Decomposing "social origins": The effects of parents' class, status, and education on the educational attainment of their children. European Sociological Review 29(5): 1024-1039.

Busset, Thomas. 1993. Zur Geschichte der eidgenössischen Volkszählung. Neuenburg: Bundesamt für Statistik.

DiPrete, Thomas A. und Claudia Buchmann. 2013. The Rise of Women. The Growing Gender Gap in Education and What it Means for American Schools. New York: Russell Sage Foundation.

Erikson, Robert und John H. Goldthorpe. 1992. The Constant Flux: A Study of Class Mobility in Industrial Societies. Oxford: Clarendon Press.

Franzen, Axel, Anna Hecken und Christoph Kopp. 2004. Bildungsexpansion und die geschlechtsspezifische Segregation an Schweizer Hochschulen. Soziale Welt 55(3): 317-335.

Glauser, David und Rolf Becker. 2015. Geschlechtsspezifische Berufswünsche und Ausbildungsentscheidungen. S. 21-47 in Berufliche Passagen im Lebenslauf, hrsg. Von Kurt Häfeli, Markus Neuenschwander und Stephan Schumann. Wiesbaden: Springer Fachmedien.

Hadjar, Andreas und Joël Berger. 2010. Dauerhafte Bildungsungleichheiten in Westdeutschland, Ostdeutschland und der Schweiz: Eine Kohortenbetrachtung der Ungleichheitsdimensionen soziale Herkunft und Geschlecht. Zeitschrift für Soziologie 39(3): 182-201.

Hill, Paul B. und Johannes Kopp. 2013. Familiensoziologie. Grundlagen und theoretische Perspektiven. Wiesbaden: Springer VS.

Hinz, Thomas und Karin Auspurg. 2011. Gruppenvergleiche bei Regressionen mit binären abhängigen Variablen - Probleme und Fehleinschätzungen am Beispiel von Bildungschancen im Kohortenverlauf. Zeitschrift für Soziologie 40(1): 62-73.

Jann, Ben und Benita Combet. 2012. Zur Entwicklung der intergenerationalen Mobilität in der Schweiz. Schweizerische Zeitschrift für Soziologie 38(2): 177-199.

Lepsius, Rainer M. 1979. Soziale Ungleichheit und Klassenstrukturen in der Bundesrepublik Deutschland. S. 166-209 in Klassen in der europäischen Sozialgeschichte, hrsg. von Hans-Ulrich Wehler. Göttingen: Vandenhoeck und Ruprecht.

Long, J. Scott. 1997. Regression Models for Categorical and Limited Dependent Variables. Thousand Oaks: Sage.

Mayer, Karl Ulrich. 2008. Retrospective longitudinal research: The German life history study. S. 85-106 in Handbook of Longitudinal Research: Design, Measurement and Analysis, hrsg. von Scott W. Menard. San Diego: Elsevier.

Müller, Walter. 1998. Erwartete und unerwartete Folgen der Bildungsexpansion. S. 83-112 in Die Diagnosefähigkeit der Soziologie, hrsg. von Jürgen Friedrichs, Rainer M. Lepsius und Karl Ulrich Mayer. Opladen: Westdeutscher Verlag. 
Müller, Walter und Markus Klein. 2008. Schein oder Sein: Bildungsdisparitäten in der europäischen Statistik. Eine Illustration am Beispiel Deutschlands. Schmoller's Jahrbuch. Zeitschrift für Wirtschafts- und Sozialwissenschaften 128(4): 511-543.

Pfeffer, Fabian T. 2008. Persistent inequality in educational attainment and its institutional context. European Sociological Review 24(5): 543-565.

Rieger, Andreas. 2001. Bildungsexpansion und ungleiche Bildungspartizipation am Beispiel der Mittelschulen im Kanton Zürich, 1830 bis 1980. Schweizerische Zeitschrift für Bildungswissenschaften 23(1): 41-72.

Scharenberg, Katja, Melanie Rudin, Barbara Müller, Thomas Meyer und Sandra Hupka-Brunner. 2014. Ausbildungsverläufe von der obligatorischen Schule ins junge Erwachsenenalter: Die ersten zehn Jahre. Ergebnisübersicht der Schweizer Längsschnittstudie TREE, Teil I. Basel: TREE, http://www.tree. unibe.ch/ergebnisse/e305140/e305154/files305157/Scharenberg_etal_2014_Synopsis_TREE_Results_Part-I_Education_ger.pdf (22.02.2016).

Schimpl-Neimanns, Bernhard. 2000. Soziale Herkunft und Bildungsbeteiligung. Empirische Analysen zu herkunftsspezifischen Bildungsungleichheiten zwischen 1950 und 1989. Kölner Zeitschrift für Soziologie und Sozialpsychologie 52(3): 636-669.

Sheldon, George. 2005. Der berufsstrukturelle Wandel der Beschäftigung in der Schweiz 1970-2000: Ausmass, Ursachen und Folgen. Neuenburg: Bundesamt für Statistik.

Urban, Dieter. 1993. Logit-Analyse. Statistische Verfahren zur Analyse von Modellen mit qualitativen Response-Variablen. Stuttgart: Fischer. 